\title{
Complicaciones posparto del síndrome HELLP: diagnóstico post mórtem
}

\author{
Postpartum complications of HELLP syndrome: postmortem \\ diagnosis
}

\begin{abstract}
Resumen
El síndrome HELLP (del inglés Hemolytic anemia, Elevated Liver enzyme, Low Platelet count) es una complicación que afecta aproximadamente al 0,1\% a 0,6\% de los embarazos que cursan con preeclampsia. El hecho de que este síndrome se asocie a una rotura hepática es una complicación rara y potencialmente letal, con una incidencia inferior a un $2 \%$, aunque algunos autores la cifran en un $9 \%$. Se ha descrito que hasta un $80 \%$ de las pacientes en que se produce un hematoma subcapsular hepático espontáneo durante el embarazo presentan preeclampsia grave o síndrome HELLP. Si se complica con una rotura hepática, las tasas de mortalidad materna y neonatal aumentan drásticamente a un $50 \%$ y un $80 \%$, respectivamente. Presentamos el caso de una primípara de 38 años de edad, que sufrió en el posparto una rotura espontánea de un hematoma hepático secundario a un síndrome HELLP no diagnosticado clínicamente.
\end{abstract}

Palabras clave: Rotura hepática espontánea. Muerte súbita. Embarazo. Síndrome HELLP. Posparto.

\begin{abstract}
HELLP syndrome is a complication that affects approximately $0,1-0,6 \%$ of pregnancies involving preeclampsia. The association of this syndrome with a liver rupture is a rare and potentially threatening complication, with an incidence of less than $2 \%$ although some authors describe it up to a $9 \%$. It has been described that up to $80 \%$ of patients with a spontaneous hepatic subcapsular hematoma during pregnancy, is associated to severe preeclampsia or HELLP syndrome. If it is complicated with liver rupture, maternal and neonatal mortality rates increase drastically to $50 \%$ and $80 \%$, respectively. We report a case of a 38 year-old primipara, who suffered a spontaneous rupture of liver haematoma, secondary to clinically undiagnosed HELLP syndrome.
\end{abstract}

Key words: Spontaneous liver rupture. Sudden death. Pregnancy. HELLP syndrome. Postpartum.

\section{Introducción}

La preeclampsia se define como una hipertensión que aparece después de las 20 semanas de gestación y se acompaña de proteinuria. Se considera grave cuando la presión arterial sistólica es $>160 \mathrm{mmHg}$ o la diastólica es $>110 \mathrm{mmHg}$ con proteinuria, o si existe hipertensión asociada a proteinuria grave (>2 g/24 h) ${ }^{1}$.

El síndrome HELLP (hemólisis, elevación de las enzimas hepáticas [GOT >70 UI, LDH >600 UI] y trombocitopenia $[<100.000$ plaquetas $/ \mathrm{ml}]$ ) es una complicación que afecta aproximadamente al $0,1 \%$ a $0,6 \%$ de los embarazos que cursan con preeclampsia ${ }^{2}$. Supone una de las complicaciones más importantes de los casos de preeclampsia grave, entre un $4 \%$ y un $15 \%{ }^{3}$.

Se ha descrito que hasta un $80 \%$ de las pacientes en quienes se produce un hematoma subcapsular hepático espontáneo durante el embarazo presentan preeclampsia grave o síndrome de HELLP. El hecho de que este síndrome se asocie a rotura hepática es una complicación rara y potencialmente letal, con una incidencia inferior a un $2 \%{ }^{4}$, aunque algunos autores la cifran en un $9 \%$.

La tasa de mortalidad materna y neonatal relacionada con el síndrome HELLP alcanza rangos del $1 \%$ al $5 \%$ y del $7,7 \%$ al $60 \%$, respectivamente. Si

\section{Beltrán ${ }^{1}$ \\ PJ. Pérez-Jorge ${ }^{2}$ \\ MC. Martínez ${ }^{3}$}

${ }^{1}$ Unidad Docente de Medicina Legal y Forense.

Facultad de Medicina. Universidad de Córdoba. Servicio de Patología Forense.

Instituto de Medicina Legal de Córdoba.

${ }^{3}$ Servicio de Histopatología. Instituto Nacional de Toxicología y Ciencias Forenses de Sevilla.

Correspondencia: Dra. Cristina M. Beltrán Aroca Unidad Docente de Medicina Legal y Forense.

Facultad de Medicina. Universidad de Córdoba. Avda. Menéndez Pidal, s/n. 14004 Córdoba, España. E-mail: h12bearc@uco.es

Fecha de recepción: 06. NOV. 2013

Fecha de aceptación: 17. DIC. 2013 
se complica con una rotura hepática, las tasas de mortalidad aumentan drásticamente a un $50 \%$ y un $80 \%$, respectivamente ${ }^{5}$.

Presentamos a continuación un caso de rotura espontánea de un hematoma hepático, en el posparto de una primípara, secundario a un síndrome HELLP no diagnosticado clínicamente.

\section{Exposición del caso}

\section{Antecedentes}

Mujer de 38 años de edad, primigesta de 40 semanas, con antecedentes de diabetes gestacional controlada, que ingresa en el hospital por cuadro de hipertensión arterial, con cifras de 150/95 mmHg. Se realiza tratamiento con infusión intravenosa de labetalol. Tras el fracaso de la inducción del parto con prostaglandinas, se le realiza cesárea, durante la cual se produjo una hemorragia intensa por atonía, ante lo que se decide practicar una histerectomía subtotal.
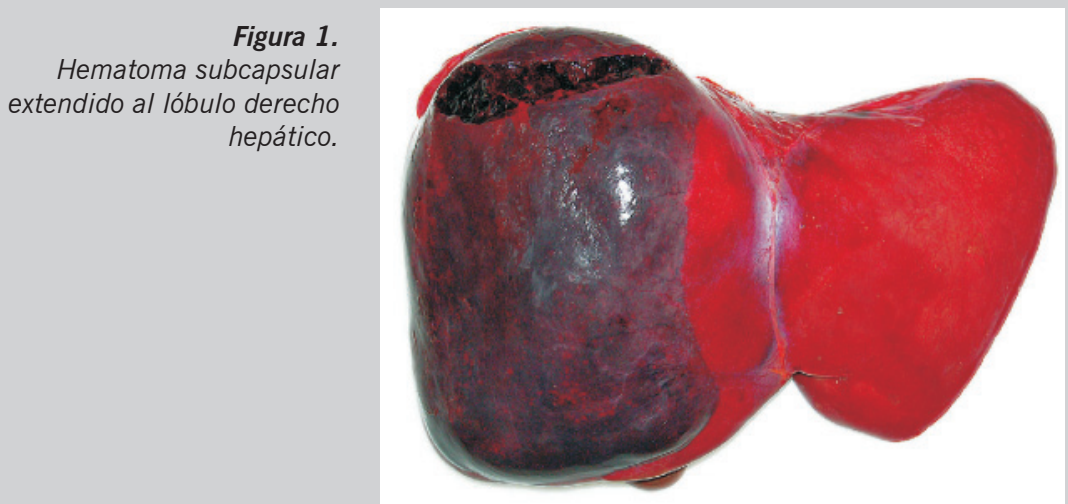

Figura 2. Imagen microscópica del parénquima hepático con importantes áreas hemorrágicas y destrucción de la arquitectura sinusoidal (HE).

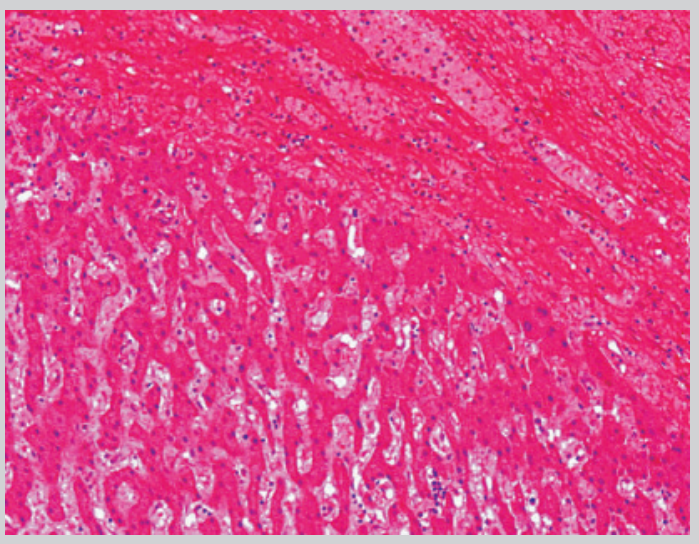

En situación de inestabilidad hemodinámica intensa y con signos de coagulopatía, ingresa en la unidad de cuidados intensivos, donde se realiza una tomografía computarizada (TC) abdomino-pélvico que informa de hematoma subcapsular hepático de 6,7 × $15 \times 19$ $\mathrm{cm}$ con áreas sugestivas de sangrado reciente, que comprime el lóbulo hepático derecho. Por indicación de cirugía se trata con medidas conservadoras, ante lo que se consigue una situación hemodinámica estable de la paciente, con disminución progresiva del hematoma subcapsular hepático según control por TC. Se realiza un análisis que pone de manifiesto: disminución del hematocrito, 18\%; bilirrubina total, 5,1 mg/dl; GOT, $815 \mathrm{U} / \mathrm{l}$; y trombocitopenia (55.000 plaquetas $/ \mathrm{ml}$ ), por lo que se procede a realizar una transfusión de plaquetas. Dada la favorable evolución clínica y ecográfica se decide el traslado de la paciente a planta, y el día anterior a recibir el alta médica, 11 días tras el parto, estando acostada y hablando con su marido, comienza a sentirse mal, con sensación disneica y pérdida de la consciencia. Tras la realización de maniobras de reanimación cardiopulmonar, la paciente fallece y se decide no certificar la defunción por muerte súbita en una enferma estable.

\section{Hallazgos de la autopsia}

En el examen externo destacaba el cadáver muy pálido con livideces poco intensas. El examen interno puso de manifiesto:

- Hemoperitoneo de sangre líquida de $950 \mathrm{~cm}^{3}$.

- Hígado aumentado de tamaño y peso, de 2.390 gramos, que presentaba un desgarro de la cápsula de Glisson en la porción superior de la cara anterior de los segmentos VII y VIII del lóbulo derecho (Figura 1).

- Gran hematoma subcapsular extendido a casi la totalidad de la cara superior y posterior del lóbulo derecho hepático (Figura 1), con dimensiones aproximadas de $22 \mathrm{~cm}$ de diámetro longitudinal, $15 \mathrm{~cm}$ de diámetro transversal y unos $3 \mathrm{~cm}$ de espesor máximo.

- Palidez visceral generalizada.

Se remitió el hígado completo para estudio histopatológico, evidenciándose un parénquima hepático muy alterado con destrucción de la arquitectura normal (Figura 2), necrosis periportal e intraparenquimatosa (Figura 3), y depósito de fibrina intrasinusoidal (Figura $4)$. Todos los hallazgos son compatibles con una rotura espontánea del hígado asociada probablemente a un síndrome HELLP. 
Por tanto, se estableció como causa de la muerte un shock hemorrágico secundario a una rotura espontánea hepática, provocada por un hematoma subcapsular en el contexto de un síndrome HELLP.

\section{Discusión}

Algunos autores describen casos atípicos de preeclampsia: antes de las 20 semanas de gestación o con signos y síntomas típicos (como aumento de peso por edemas, cefalea, dolor epigástrico o en el hipocondrio derecho, alteraciones visuales) sin hipertensión o proteinuria ${ }^{6}$, y de síndrome $\mathrm{HELLP}^{7}$ sin alteraciones analíticas que, al igual que en nuestro caso, dificulta el diagnóstico.

Aunque la preeclampsia y el síndrome HELLP son más frecuentes en las mujeres primíparas, se ha descrito clásicamente que el riesgo de rotura hepática espontánea es mayor en las multíparas entre 28 y 35 años de edad ${ }^{8,9}$. Sin embargo, la paciente del caso referido supera los 35 años de edad, aunque es primípara, algo ya recogido por un estudio realizado 4 que puso de manifiesto una mayoría de mujeres primíparas menores de 30 años que presentaron esta complicación, explicando los autores estas variaciones por las diferencias socioculturales de cada país.

En la literatura se recoge ampliamente como factor predisponente de la rotura de un hematoma hepático, la presencia de preeclampsia o de síndrome HELLP durante el embarazo, pero también algunos autores han descrito casos en los que se presenta aisladamente $\mathrm{i}^{10-12}$. Destacamos que en nuestro caso la paciente tuvo un embarazo de evolución normal, con diabetes gestacional controlada, parámetros analíticos y cifras tensionales dentro de la normalidad, y al final de la gestación presentó un episodio de hipertensión arterial aislado que justificó su ingreso y la inducción del parto.

Tras realizar la cesárea, complicada debido a una atonía uterina, la paciente presenta un intenso dolor abdominal con signos de coagulopatía, trombocitopenia e inestabilidad hemodinámica, diagnosticándose un hematoma subcapsular hepático. Generalmente, el hematoma hepático y la rotura espontánea en pacientes con síndrome HELLP ocurren en el tercer trimestre o a finales del segundo, pero es importante recordar que el riesgo de rotura hepática no se reduce tras el parto o la extracción urgente del feto, ya que puede presentarse 24 a 48 horas después del parto o la completa regresión de este síndrome ${ }^{4,13}$. En embarazos con evolución normal y con un parto normal

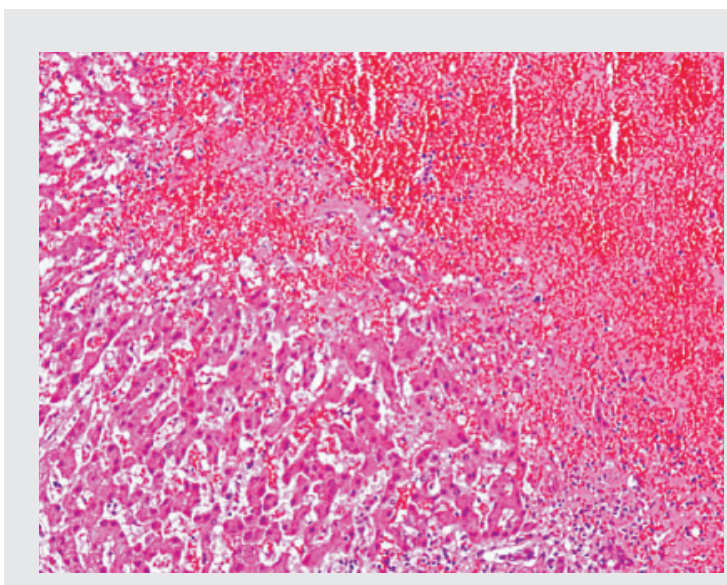

Figura 3.

Imagen microscópica en la que se observan áreas hemorrágicas, infiltrado inflamatorio y necrosis periportal e intraparenquimatosa (HE).

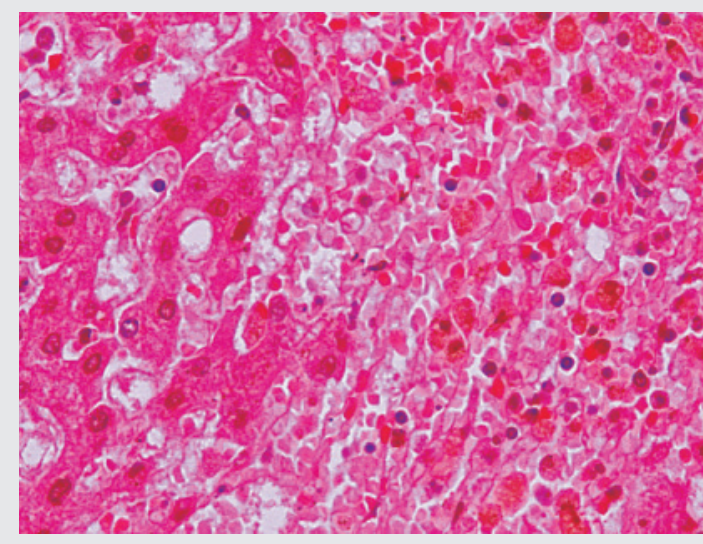

Figura 4.

Microscópicamente se aprecian macrófagos cargados de hemosiderina y tramas de fibrina (HE). también se han referido casos en los que sucede en el período inmediato al posparto ${ }^{14}$ e incluso hasta 3 días después ${ }^{12}$.

Las medidas adoptadas son conservadoras, debido a la espontánea resolución del hematoma, pero a pesar de la favorable evolución clínica y ecográfica, 11 días tras el parto la paciente muere súbitamente. La autopsia revela el hematoma subcapsular y el desgarro en la cápsula de Glissom junto a un importante hemoperitoneo.

No se conoce con exactitud la causa última que conduce a la rotura hepática, aunque el informe histopatológico pone de manifiesto hallazgos histológicos que indican que pueden desempeñar un papel fundamental la hemorragia periportal y el depósito intravascular de fibrina en el hígado. Esto conduciría a una obstrucción de los sinusoides, provocando un aumento de la presión intrahepática debido a la congestión vascular. Se produciría así una necrosis hepática y una hemorragia intraparenquimatosa y 
subcapsular. En los casos más graves podría, finalmente, provocar una rotura de la cápsula ${ }^{13}$.

Como conclusión, se trata de un caso poco frecuente y por ello resulta necesario un estudio exhaustivo de los hallazgos post mórtem junto a la historia clínica, indagando en los factores etiopatogénicos implicados y teniendo en cuenta las posibles variaciones en su presentación clínica, ya que puede pasar desapercibido para el médico. Así será posible conocer la incidencia y la prevalencia de síndromes poco frecuentes con consecuencias fatales, y podrá establecerse una adecuada correlación clínico-patológica para determinar las causas de la muerte. Además, ayudará a aclarar aquellos casos en que se sospeche que la muerte ha podido ser resultado de una mala praxis médica, así como también prevenir las posibles denuncias ante los tribunales.

Los autores declaran no tener conflicto de intereses.

\section{Bibliografía}

1. Protocolos SEGO. Prog Obstet Ginecol. 2007;50(7): 446-55.

2. Santos-Bolívar J, et al. Ruptured subcapsular hepatic haematoma: a HELLP syndrome complication. Cir Esp. 2010;87(1):50-1.

3. Gutiérrez-Cafranga E, et al. Hepatic rupture and hemoperitoneum in a pregnant woman with HELLP syndrome. Rev Esp Enferm Dig. 2010;102(7):4534.

4. Grand'Maison S, et al. Hepatic rupture in hemolysis, elevated liver enzymes, low platelets syndrome. Obstet Gynecol. 2012;119(3):617-25.

5. Kulungowski AM, et al. Hemolysis, elevated liver enzymes, and low platelets syndrome: when is surgical help needed? Am J Surg. 2009;198(6):916-20.

6. Stella CL, Malik KM, Sibai BM. HELLP syndrome: an atypical presentation. Am J Obstet Gynecol. 2008; 198(5).

7. Sibai BM. Diagnosis, controversies, and management of the syndrome of hemolysis, elevated liver enzymes, and low platelet count. Obstet Gynecol. 2004;103(5 Pt 1):981-91.
8. Araujo AC, et al. Characteristics and treatment of hepatic rupture caused by HELLP syndrome. Am J Obstet Gynecol. 2006;195:129-33.

9. Vigil-De Gracia P, Ortega-Paz L. Pre-eclampsia/ eclampsia and hepatic rupture. Int J Gynaecol Obstet. 2012;118(3):186-9.

10. Shakya VC, et al. An alarming but self-limited case of isolated large spontaneous liver hematoma in pregnancy. Pan Afr Med J. 2013;14:36.

11. Sutton BC, et al. Fatal postpartum spontaneous liver rupture: case report and literature review. J Forensic Sci. 2008;53(2):472-5.

12. Shaw C, et al. Spontaneous rupture of the liver following a normal pregnancy and delivery. Ir Med J. $2005 ; 98(1): 27-8$.

13. Pavlis T, et al. Diagnosis and surgical management of spontaneous hepatic rupture associated with HELLP syndrome. J Surg Educ. 2009;66(3):163-7.

14. Abdi S, et al. Spontaneous hepatic rupture and maternal death following an uncomplicated pregnancy and delivery. BJOG. 2001;108(4):431-3. 